\title{
Successful Treatment of Severe Parainfluenza Pneumonia in a Liver Transplant Recipient with Oral Ribavirin and Intravenous Immunoglobulin
}

\author{
Fereshteh Ghiasvand ${ }^{1}$, Zahra Ahmadinejad ${ }^{1}$, Davood Mansouri ${ }^{2}$, Elham Raisi $^{3}$, Mohammad Taher $^{4}$ \\ and Samrand Fattah Ghazi ${ }^{5,{ }^{*}}$ \\ ${ }^{1}$ Liver transplantation Research Center, Department of Infectious Diseases, Imam Khomeini Hospital Complex, Tehran University of Medical Sciences, Tehran, Iran \\ ${ }^{2}$ Department of Infectious Diseases and Clinical Immunology, National Research Institute of Tuberculosis and Lung Diseases, Masih Daneshvary Hospital, Shahid Beheshti \\ University of Medical Sciences, Tehran, Iran \\ ${ }^{3}$ Batman, Western Australia \\ ${ }^{4}$ Department of Gastroenterology and Hepatology, Imam Khomeini Hospital Complex, Tehran University of Medical Sciences, Tehran, Iran \\ ${ }^{5}$ Department of Anesthesiology and Critical Care, Imam Khomeini Hospital Complex, Tehran University of Medical Sciences, Tehran, Iran \\ "Corresponding author: Department of Anesthesiology and Critical Care, Imam Khomeini Hospital Complex, Tehran University of Medical Sciences, Tehran, Iran. Email: \\ samrand1@gmail.com
}

Received 2020 January 04; Revised 2020 September 29; Accepted 2020 November 15.

\begin{abstract}
The most common viruses affecting immunocompromised hosts include cytomegalovirus, herpes simplex virus, varicella-zoster virus, influenza virus, measles virus, and adenovirus. Parainfluenza virus infection is common among human populations. It causes non-specific symptoms in immune-competent hosts; therefore, it may go undiagnosed. However, it is particularly dangerous for immunocompromised patients by presenting a high risk of morbidity and sometimes mortality among them. Patients who have bone marrow or solid organ transplantation need to follow an intense immunosuppressing routine, which leaves them extremely vulnerable to opportunistic agents such as parainfluenza viruses. Several factors determine the severity of parainfluenza virus infections, such as the type of the virus, the level of immunosuppression, and co-infection with other agents. Co-infection is especially important because it makes a correct differential diagnosis difficult. While almost all bodies of influenza infection information in immunocompromised patients are from studies of bone marrow transplant recipients, there are very limited data available on influenza infections in liver transplant patients. We present a liver transplant patient with severe parainfluenza pneumonia that was improved significantly with oral ribavirin and intravenous immunoglobulin. A 23-year-old female patient with a history of liver transplantation from 18 months ago presented with a one-week cough and tachypnea. Chest computed tomography demonstrated ground-glass opacities and diffuse infiltrates throughout both lungs. A multiplex polymerase chain reaction for the detection of respiratory viruses in the nasopharynx was positive for parainfluenza virus type 3 and she was successfully treated with oral ribavirin and intravenous immunoglobulin. Respiratory infection with the parainfluenza virus in immunocompromised adults, including transplant patients, is associated with significant morbidity and mortality, and early treatment with ribavirin and intravenous immunoglobulin may markedly improve their outcomes. To the best of our knowledge, this is the first case report of the successful treatment of parainfluenza infection with ribavirin and intravenous immunoglobulin in a patient with liver transplantation.
\end{abstract}

Keywords: Parainfluenza, Liver Transplant, Ribavirin

\section{Introduction}

Parainfluenza viruses (subtypes 1 - 4) are singlestranded RNA viruses and the members of the Paramyxoviridae family. Parainfluenza virus types 1 and 2 characteristically cause epidemic diseases in the fall, while type 3 produces diseases throughout the year $(1,2)$. The initial symptoms are non-specific and include cough, fever, dyspnea, and sputum production (3). Initiating antiviral therapy, especially at the early stages of infection, has been reported in case series to reduce the risk of progression to pneumonia and improve the prognosis. Ribavirin is a nucleoside analog with broad-spectrum antiviral effects against RNA viruses. It affects the viral genome and increases the mutation rate of RNA, resulting in virus death. When there is evidence of upper or lower respiratory tract infection, in the right clinical setting, the parainfluenza virus should be suspected and rapidly confirmed by the identification of the virus in nasopharyngeal secretions 
via viral culture, molecular assays, polymerase chain reaction (PCR), or fluoro-immunoassays (4).

As infection with the parainfluenza virus can cause non-specific symptoms in immune-competent hosts, it may go undiagnosed (5). However, it is particularly dangerous for immunocompromised patients by presenting a high risk of morbidity and sometimes mortality among them. Parainfluenza virus infection is associated with significant morbidity and sometimes mortality in immunocompromised patients, including the recipients of marrow or solid organ transplants (6). Several factors determine the severity of parainfluenza virus infections, such as the type of the virus, the level of immunosuppression, and co-infection with other agents. Co-infection is especially important because it makes a correct differential diagnosis difficult.

There are some reports that parainfluenza pneumonia in solid organ transplants results in high mortality despite ribavirin use $(7,8)$. While almost all bodies of influenza infection information in immunocompromised patients are from studies of bone marrow transplant recipients, there are very limited data available on influenza infections in liver transplant patients. In this report, we present a liver transplant patient with severe parainfluenza pneumonia that was improved significantly with oral ribavirin and intravenous immunoglobulin (IVIG).

\section{Case Presentation}

A 23-year-old female patient presented with a one-week history of cough and tachypnea in December 2018. The patient did not have myalgia, sore throat, or rhinorrhea. The past medical history included liver transplantation 18 months ago due to Budd-Chiari syndrome, which subsequently led to three pulse methylprednisolone injections four months ago due to acute rejection. Her immunosuppressive regimen at the time of admission was prednisolone $20 \mathrm{mg}$ daily, mycophenolate sodium $360 \mathrm{mg}$ daily, and tacrolimus $1 \mathrm{mg}$ daily. She had not received an influenza vaccine in the previous autumn season, and there was no history of similar symptoms in other members of her family. On the first day of admission, she was febrile $\left(38.5^{\circ} \mathrm{C}\right)$ with severe dyspnea. $\mathrm{Her}_{2}$ saturation in room air was $70 \%$, blood pressure was $120 / 75 \mathrm{mmHg}$, and the respiratory rate was 28 - 30 per minute. Furthermore, her physical examinations were normal, except for significant basilar rales in the lungs.

No gross abnormality was reported on the chest X-ray. Chest computed tomography (CT) demonstrated groundglass opacities, and diffuse infiltrates throughout both lungs (Figure 1). She was transferred immediately to the
Intensive Care Unit (ICU) but was not intubated. Supportive care, such as cardiac monitoring and oxygen therapy started immediately. Initial antimicrobial treatment comprised of intravenous (IV) meropenem ( $2 \mathrm{~g}$ three times a day), vancomycin (1 $\mathrm{g}$ twice a day), and levofloxacin (750 mg daily) to cover bacterial pneumonia, besides a double dose of oseltamivir because of high suspicion of influenza.

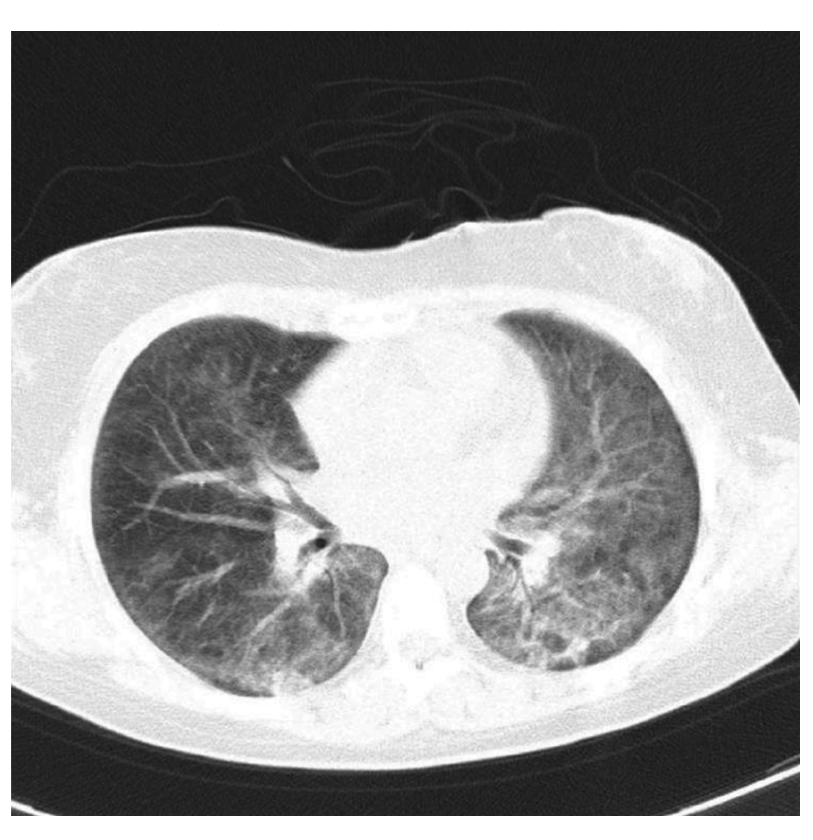

Figure 1. Computed tomography scan of the chest obtained after admission before treatment with oral ribavirin and intravenous immunoglobulin (IVIG)

Upon further deterioration of the patient's condition on the second day of admission, co-trimoxazole was administered to cover Pneumocystis jiroveci pneumonia (PCP), as well as ganciclovir to cover cytomegalovirus (CMV) pneumonitis. Later on, amphotericin B was added to the regimen to cover fungal infection. The diagnostic tests were done, including multiplex PCR for the detection of respiratory viruses in the nasopharynx, blood cultures, sputum smear, and culture for bacterial, mycobacterial, and fungal infections. A sputum sample was also tested for Pneumocystis jiroveci by PCR. Moreover, the patient was a candidate for bronchoscopy and Broncho-alveolar lavage (BAL), but ultimately it was aborted due to adverse clinical conditions and potential risk of hemorrhage in BuddChiari syndrome.

During the subsequent three days, the patient deteriorated clinically and developed signs of respiratory failure. A repeated chest CT scan showed extensive changes in bilateral ground-glass opacities (Figure 2). Considering that she was awake and her hemodynamic parameters were acceptable, and arterial oxygen saturation by an oxygen 
mask and reservoir bag was $92 \%$, it was decided not to intubate the patient. Nevertheless, the ventilator was kept on standby to avoid hypoxia (i.e., $\mathrm{SPO}_{2}<90 \%$ ) or fatigue of respiratory muscles. On the seventh day, the multiplex PCR of nasopharynx swab was reported positive for parainfluenza virus type 3 by two different laboratory settings. Furthermore, the PCR result for Pneumocystis jiroveci was reported negative. A weak band of CMV was reported by one of the laboratory settings, too (Table 1) that had a doubtful significance, and serum CMV PCR was negative. All other investigation results were negative, and blood cultures yielded no growth.

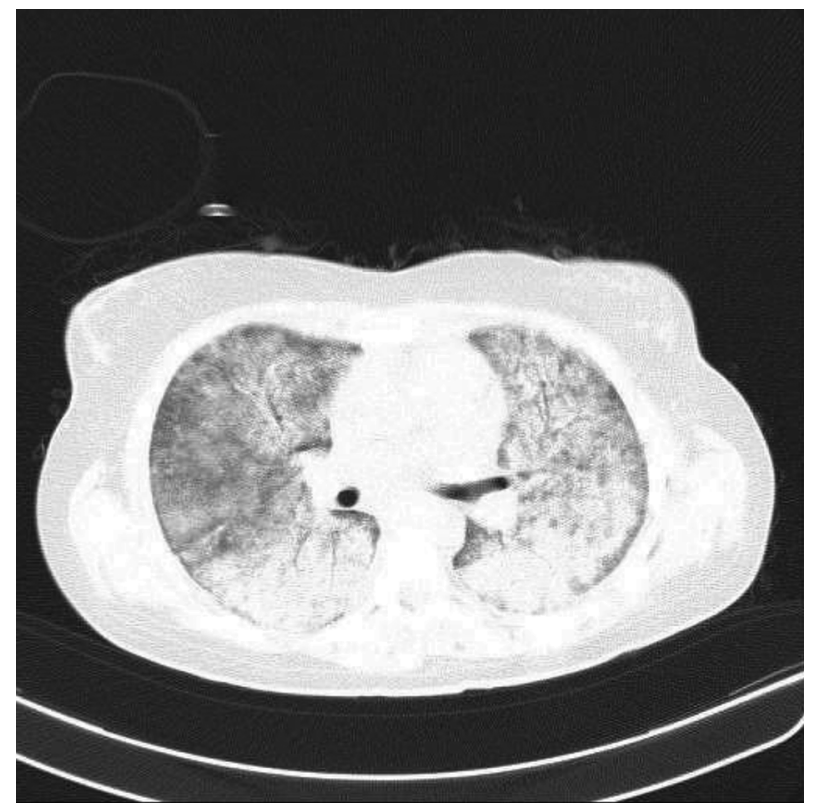

Figure 2. Chest computed tomography scan reveals extensive changes of bilateral ground-glass opacities after three days

Considering the poor response to different broadspectrum drug regimens and the laboratory findings, we decided to change the treatment course. On the seventh day of admission, the administration of meropenem, vancomycin, amphotericin, and oseltamivir was ceased consecutively from day seven until day 10. Simultaneously, we started oral ribavirin $20 \mathrm{mg} / \mathrm{kg} /$ day and intravenous immunoglobulin (IVIG) $20 \mathrm{~g} /$ day (for four days). Then, 24 hours later, IV dexamethasone $4 \mathrm{mg}$ three times a day was added to the treatment plan. She became afebrile within 48 hours and improved clinically in the next days. Ribavirin continued for 14 days, while dexamethasone was given for seven days. The co-trimoxazole treatment course was completed, and ganciclovir continued for 21 days. The patient showed a dramatic clinical improvement and did

\begin{tabular}{ll}
\hline Table 1. Microbiological Tests & \\
\hline Tests & Sputum PCR \\
\hline Parainfluenza-3 & Positive \\
\hline Adenovirus & Negative \\
\hline CMV & Positive \\
\hline Influenza A & Negative \\
\hline Influenza B & Negative \\
\hline Parainfluenza-1 & Negative \\
\hline Parainfluenza-2 & Negative \\
\hline Respiratory syncytial virus & Negative \\
\hline Acid-fast bacilli & Negative \\
\hline Fungi & Negative \\
\hline Pneumocystis jiroveci & Negative \\
\hline Coronavirus & Negative \\
\hline Ab & \\
\hline
\end{tabular}

Abbreviations: CMV, Cytomegalovirus; PCR, polymerase chain reaction.

not require intubation nor further stay in the ICU, and was transferred to the general respiratory ward.

After the complete ribavirin course, she did not have crepitation in her lung bases. Oxygen saturation was normalized in room air, and chest X-ray showed near-complete resolution of previous widespread infiltrates. We performed daily complete blood counts to check for possible anemia normally attributed to high doses of ribavirin, and the results did not show a drop in hemoglobin. However, as the patient was receiving ganciclovir, she developed leukopenia (a known side effect of ganciclovir) during her hospital stay, which was treated with two doses of granulocyte colony stimulating factor (GCSF). Within two weeks, post-commencing ribavirin, the patient's respiratory function returned to normal baseline and remained normal on the follow-up session post-discharge. Similarly, her oxygen saturation remained $95 \%$ in room air. In the follow-up appointment two months later, the chest CT scan revealed near-normal lung parenchyma.

\section{Discussion}

The most common viruses affecting immunocompromised hosts include cytomegalovirus, herpes simplex virus, varicella-zoster virus, influenza virus, measles virus, and adenovirus (1). The outcome of infection is poor with the respiratory syncytial virus and parainfluenza 3 in allogeneic hematopoietic transplant patients. The initial upper respiratory tract infection often progresses to lower respiratory tract infection, with the development of respiratory distress syndrome and/or respiratory failure $(9,10)$. Initiating antiviral therapy, especially at the early stages 
of infection, has been reported in case series to reduce the risk of progression to pneumonia and improve the prognosis. Ribavirin is a nucleoside analog with broadspectrum antiviral effects against RNA viruses. It affects the viral genome and increases the mutation rate of RNA, resulting in virus death. Ribavirin has been previously approved for use in viral hemorrhagic fever (11).

There are some studies regarding the efficacy of ribavirin in the treatment of parainfluenza viruses. In a 2,700bed tertiary care hospital in Seoul, South Korea, from January 2009 to February 2012, Park et al. (7) identified patients who were infected with respiratory viruses and evaluated the efficacy of oral ribavirin in patients with hematologic disease. They suggested that oral ribavirin therapy may not improve clinical outcomes in patients with the hematologic disorder infected with paramyxovirus (7). In contrast, Shima et al. (12) reported a case of a bone marrow transplant recipient with parainfluenza virus 3 pneumonia that was treated successfully with oral ribavirin in 2008. Falsey (8) had a review in 2012 about the management of parainfluenza pneumonitis in immunocompromised patients. This review concluded that the use of aerosolized or systemic ribavirin in combination with intravenous gamma globulin had been reported in small, uncontrolled series, and case reports of immunocompromised patients. The majority of the data were in hematopoietic stem cell transplantation (HSCT) patients, and the consensus indicated that if ribavirin was given late in the course of illness, it would not be effective for parainfluenza pneumonia, especially in patients with respiratory failure. This review revealed that some reports suggest the modest benefit of ribavirin at the early stage of upper respiratory tract involvement, but this is controversial because of the lack of controlled trials (8).

In our patient, considering multiple factors such as negative results of PCP and other respiratory viruses, the confirmation of parainfluenza virus type 3 in two different laboratory settings, only one positive report of a weak band of CMV, and the significant clinical response to ribavirin, so it seemed that parainfluenza pneumonitis was the most probable diagnosis. Ribavirin was an effective treatment for her despite respiratory failure and the risk of high mortality of parainfluenza infection in transplant patients. In addition to antiviral therapy, corticosteroids and IVIG may play an effective role in the management of patients with respiratory distress syndrome secondary to parainfluenza infection (13-15). We also noted a significant clinical response to IV dexamethasone and IVIG in our patient. Respiratory infection with the parainfluenza virus in immunocompromised adults, including transplant patients, is associated with significant morbidity and mortality, and early treatment with ribavirin and IVIG may sig- nificantly improve their conditions. Successful treatment of parainfluenza infection with ribavirin and intravenous immunoglobulin in a patient with a liver transplant has rarely been reported $(16,17)$

\section{Footnotes}

Authors' Contribution: DM developed the original idea. FG, ER, and ZA abstracted and wrote the manuscript. MT and SGH contributed to manuscript preparation.

Conflict of Interests: All authors declare that they have no relevant financial interests and no conflict of interest.

Funding/Support: This manuscript was fully sponsored by the Tehran University of Medical Sciences.

Informed Consent: Informed Consent was signed by the patient.

\section{References}

1. Greenberg SB. Viral pneumonia. Infect Dis Clin North Am. 1991;5(3):60321. [PubMed:1659594].

2. Heilman CA. From the National Institute of Allergy and Infectious Diseases and the World Health Organization. Respiratory syncytial and parainfluenza viruses. J Infect Dis. 1990;161(3):402-6. doi: 10.1093/infdis/161.3.402. [PubMed: 2155971].

3. Falsey AR, Betts RF. Viral pneumonia: still a force to be reckoned with. J Respir Dis. 1993;14:31.

4. Hierholzer JC, Bingham PG, Coombs RA, Johansson KH, Anderson LJ, Halonen PE. Comparison of monoclonal antibody timeresolved fluoroimmunoassay with monoclonal antibody capturebiotinylated detector enzyme immunoassay for respiratory syncytial virus and parainfluenza virus antigen detection. J Clin Microbiol. 1989;27(6):1243-9. doi: 10.1128/JCM.27.6.1243-1249.1989. [PubMed: 2546973]. [PubMed Central: PMC267535].

5. Wendt $\mathrm{CH}$, Weisdorf DJ, Jordan MC, Balfour HJ, Hertz MI. Parainfluenza virus respiratory infection after bone marrow transplantation. $N$ Engl $J$ Med. 1992;326(14):921-6. doi: 10.1056/NEJM199204023261404. [PubMed: 1311800].

6. Russell E, Yang A, Tardrew S, Ison MG. Parainfluenza Virus in Hospitalized Adults: A 7-Year Retrospective Study. Clin Infect Dis 2019;68(2):298-305. doi: 10.1093/cid/ciy451. [PubMed: 29961826].

7. Park SY, Baek S, Lee SO, Choi SH, Kim YS, Woo JH, et al. Efficacy of oral ribavirin in hematologic disease patients with paramyxovirus infection: analytic strategy using propensity scores. Antimicrob Agents Chemother. 2013;57(2):983-9. doi: 10.1128/AAC.01961-12. [PubMed: 23229488]. [PubMed Central: PMC3553680].

8. Falsey AR. Current management of parainfluenza pneumonitis in immunocompromised patients: a review. Infect Drug Resist. 2012;5:1217. doi: 10.2147/IDR.S25874. [PubMed: 22893749]. [PubMed Central: PMC3418768].

9. Lewis VA, Champlin R, Englund J, Couch R, Goodrich JM, Rolston K, et al. Respiratory disease due to parainfluenza virus in adult bone marrow transplant recipients. Clin Infect Dis. 1996;23(5):1033-7. doi: 10.1093/clinids/23.5.1033. [PubMed: 8922798].

10. Nichols WG, Gooley T, Boeckh M. Community-acquired respiratory syncytial virus and parainfluenza virus infections after hematopoietic stem cell transplantation: the Fred Hutchinson Cancer Research Center experience. Biol Blood Marrow Transplant. 2001;7 Suppl:11S-5S. doi:10.1053/bbmt.2001.v7.pm11777098. [PubMed: 11777098]. 
11. Montaner JS, Lawson LM, Levitt N, Belzberg A, Schechter MT, Ruedy J Corticosteroids prevent early deterioration in patients with moderately severe Pneumocystis carinii pneumonia and the acquired immunodeficiency syndrome (AIDS). Ann Intern Med. 1990;113(1):14-20. doi: 10.7326/0003-4819-113-1-14. [PubMed: 2190515].

12. Shima T, Yoshimoto G, Nonami A, Yoshida S, Kamezaki K, Iwasaki H, et al. Successful treatment of parainfluenza virus 3 pneumonia with oral ribavirin and methylprednisolone in a bone marrow transplant recipient. Int J Hematol. 2008;88(3):336-40. doi: 10.1007/s12185-0080148-6. [PubMed: 18712461].

13. Bozzette SA, Sattler FR, Chiu J, Wu AW, Gluckstein D, Kemper C, et al. A controlled trial of early adjunctive treatment with corticosteroids for Pneumocystis carinii pneumonia in the acquired immunodeficiency syndrome. N Engl J Med.1990;323(21):1451-7.

14. Gagnon S, Boota AM, Fischl MA, Baier H, Kirksey OW, La Voie L. Corticosteroids as adjunctive therapy for severe Pneumocystis carinii pneumonia in the acquired immunodeficiency syndrome. A doubleblind, placebo-controlled trial. NEngl J Med.1990;323(21):1444-50. doi: 10.1056/NEJM199011223232103. [PubMed: 2233916].

15. Cotugno N, Manno EC, Stoppa F, Sinibaldi S, Saffirio C, D’Argenio P, et al. Severe parainfluenza pneumonia in a case of transient hypogammalobulinemia of infancy. BMJ Case Rep. 2013;2013. doi: 10.1136/bcr2013-009959. [PubMed: 23814123]. [PubMed Central: PMC3703058].

16. Sridhar S, Luk HK, Lau SK, Woo PC. First report of severe parainfluenza virus $4 \mathrm{~B}$ and rhinovirus $\mathrm{C}$ coinfection in a liver transplant recipient treated with immunoglobulin. J Clin Virol. 2014;61(4):611-4. doi: 10.1016/j.jcv.2014.09.019. [PubMed: 25453574].

17. Herzog KD, Dunn SP, Langham MJ, Marmon LM. Association of parainfluenza virus type 3 infection with allograft rejection in a liver transplant recipient. Pediatr Infect Dis J. 1989;8(8):534-6. [PubMed: 2549495]. 